\title{
Dietas de Baixo Teor de Carboidrato no Tratamento do Diabetes Mellitus Tipo 2
}

\author{
Marcela Cristina de Andrade, ${ }^{1}$ Fabíola Pansani Maniglia ${ }^{2}$
}

\begin{abstract}
RESUMO
A dieta de baixo consumo de carboidratos (do inglês low carb) vem sendo largamente estudada para auxiliar no manejo do diabetes mellitus tipo 2. Os resultados demostram melhor controle da glicose sanguínea. 0 objetivo deste trabalho foi analisar resultados de ensaios clínicos que utilizaram dietas de baixo consumo de carboidratos, em comparação às dietas tradicionais aconselhadas no tratamento do diabetes mellitus tipo 2, quanto aos marcadores bioquímicos de melhora do controle da doença, redução ou exclusão do uso de medicamentos, diminuição da circunferência da cintura e perda de massa corporal. Os artigos foram selecionados por meio de pesquisa bibliográfica na base de dados Pubmed, cumprindo os seguintes critérios: ensaios clínicos randomizados utilizando a dieta low carb, publicados no período de 1998 a 2018 em português, espanhol ou inglês. Considerou-se como dieta de baixo teor de carboidrato aquelas que ofereciam percentuais do macronutriente iguais ou inferiores a $45 \%$ ou menos de $130 \mathrm{~g}$ por dia. Os resultados dos 14 artigos incluídos nesta revisão apresentaram diferença na perda de massa corporal e diferença expressiva nas reduções de hemoglobina glicada, glicemia de jejum, triglicérides e redução do uso de medicamentos, em favor das dietas low carb. Esta revisão demonstra que uma dieta com baixo teor de carboidratos é efetiva no controle dos marcadores do diabetes mellitus tipo 2, podendo o paciente ter uma melhor qualidade de vida por meio da alimentação, embora mais estudos de longo prazo e de amostra aumentada sejam necessários para apoiar esta dieta como tratamento da doença.
\end{abstract}

Palavras-chave: Diabetes mellitus tipo 2. Dieta de baixo teor de carboidratos. Hemoglobina glicada.

\section{LOW CARBOHYDRATE DIETS IN TYPE 2 DIABETES TREATMENT}

\section{ABSTRACT}

A low carbohydrate diet (low carb) is widely studied to manage type 2 diabetes, demonstrating better blood glucose control can be achieved with a lower carbohydrate intake. The aim of this study was to analyze the results of clinical trials that used low carbohydrate diets in comparison to the traditional diets recommended in type 2 diabetes treatment, as for the biochemical markers of improvement disease control, reduction or exclusion of medicines usage, reduction of the waist circumference and weight loss. The articles were selected by bibliographic research in the Pubmed database, fulfilling the following criteria: randomized clinical trials using the low carb diet, published between 1998 and 2018 in Portuguese, Spanish or English. It was considered as a low carbohydrate diet, those offer macronutrient percentages equal or less than $45 \%$ or less than $130 \mathrm{~g}$ per day. The results of the 14 articles included in this review showed difference in weight loss and a significant difference in reductions in glycated hemoglobin, fasting glucose, triglycerides and reduction of drug use, in favor of low carb diets. This review demonstrates that a low carbohydrate diet is effective in controlling the markers of type 2 diabetes, and the patient may have a better quality of life through diet. Although, more long-term and increased sample studies are needed to support this diet as treatment of the disease.

Keywords: Type 2 Diabetes. Low carbohydrate diet. Glycated hemoglobin. 


\section{INTRODUÇÃO}

Incialmente, o diabetes mellitus era diferenciado em duas formas pelos termos "dependente de insulina" e "não dependente de insulina". Atualmente, as definições são classificadas etiologicamente por diabetes mellitus tipo 1, tipo 2 e gestacional, sendo essas as três formas mais conhecidas (SBD, 2019). A doença é uma associação de alterações metabólicas, e todos os tipos têm em comum o nível excessivo de glicose no sangue, denominado hiperglicemia. Este aumento da concentração sérica de glicose é causado por problemas decorrentes na produção ou na ação e secreção da insulina (GROSS et al., 2002).

Quando a doença se manifesta em algumas muIheres durante a gestação, ela recebe o nome de diabetes gestacional. Neste período a mulher sofre com variações hormonais, e pode apresentar uma resistência à insulina (SBD, 2018). Desta forma, há um aumento permanente do nível de glicose no sangue, que, geralmente, permanece apenas durante o período da gravidez, normalizando-se após o parto, quando o perfil hormonal volta ao normal (GROSS et al., 2002).

Outra forma da doença é o diabetes mellitus tipo 1 , que pode ter causa autoimune ou desconhecida e acomete uma menor porcentagem de indivíduos. A doença leva à destruição das células beta do pâncreas de indivíduos susceptíveis geneticamente, com a presença de anticorpos e um processo de insulite, causando ausência de células secretoras de insulina (SBD, 2018).

Já o terceiro tipo de diabetes, o tipo 2, tem sua causa ligada à predisposição genética e, especialmente, aos maus hábitos de vida, que estão relacionados ao aumento da resistência à insulina, como: alimentação inadequada, sedentarismo e ganho de massa corporal excessivo, os quais levam à progressão e instalação crônica da hiperglicemia (ADA, 2014). O último registro da International Diabetes Federation (IDF) feito em 2019, indicou que 463 milhões de adultos foram diagnosticados com diabetes (IDF, 2019). Este diagnóstico pode ocorrer tardiamente, pois os sintomas e agravamentos da doença acontecem de forma gradual, com quadros de hiperglicemia recorrentes. Por esta razão, é mais comum que o diabetes mellitus tipo 2 acometa, em maior proporção, os indivíduos adultos e idosos, normalmente com sobrepeso ou obesidade e acúmulo de gordura abdominal (MCLELLAN et al., 2007). Tal perfil antropométrico é diferente daquele observado no diabetes mellitus tipo 1, em que os indivíduos majoritariamente se apresentam eutróficos ou emagrecidos (ADA, 2014).
No diabetes mellitus tipo 2, a fase inicial da doença costuma ser assintomática, pois a produção do hormônio insulina em resposta ao consumo de carboidratos, ainda é eficiente no controle da glicose sanguínea (ADA, 2014). Com o passar do tempo, ocorre uma resistência à insulina, em que o aumento excessivo da produção desta, na tentativa de compensar os níveis elevados de glicose sanguínea, estão associados aos erros na secreção e mecanismo de ação e sinalização intracelular deste hormônio (SOARES, 2018). Em alguns casos, a resistência à insulina pode progredir para a falência das células beta produtoras do hormônio em razão da sobrecarga do pâncreas. Por esta razão, em alguns casos, pode ser necessária a administração exógena de insulina. Esta terapia, no entanto, é menos comum nos casos de diabetes mellitus tipo 2 (SBD, 2019).

Uma das medidas terapêuticas para auxílio no controle da glicose e minimização de outras alterações metabólicas é a indicação de hipoglicemiantes orais. Estes medicamentos têm como finalidade manter a glicemia do paciente próxima dos valores normais de referência para uma pessoa saudável. $O$ uso da droga apenas, no entanto, não é suficiente para tratar a doença de forma efetiva, sendo necessário, também, garantir a estes indivíduos uma educação nutricional, com mudanças no comportamento alimentar. A dietoterapia é fundamental no controle da doença, sendo um tratamento nutricional de primeira escolha, aliado à prática de exercício físico (SBD, 2019).

As principais recomendações nutricionais de centros de tratamento de diabetes, como a Joslin Diabetes Center, tratam da exclusão do açúcar puro e da modificação das fontes alimentares de carboidratos (JOSLIN DIABETES CENTER, 2018). Acredita-se que com um consumo mais baixo de carboidratos simples e refinados, evitar-se-ia os picos glicêmicos (BASU et al., 2013). Tais recomendações assemelham-se àquelas realizadas em outros tipos de intolerância alimentar, eliminando o alimento ou a substância que não é tolerada pelo organismo (SBP, 2008).

Em termos numéricos, a American Diabetes Association (ADA), associação que elabora as principais condutas no tratamento do diabetes, recomenda uma ingestão de carboidratos de cerca de $45-50 \%$ do total de calorias consumidas, com déficit de 500 calorias/ dia para a redução ponderal (ADA, 2017). Os estudos têm mostrado, no entanto, a baixa eficácia destas orientações para o controle metabólico do diabetes (VOLEK et al., 2008). A ADA, juntamente com a Associação Europeia para o Estudo do Diabetes, publicaram, no ano de 2018, um relatório de consenso em fa- 
vor da utilização de estratégias de dietas low carb para controle da hiperglicemia em pacientes com diabetes mellitus tipo 2 (DAVIES et al., 2018). Por este motivo, pesquisadores decidiram testar os efeitos de dietas de baixo teor de carboidrato, com percentual de ingestão igual ou menor que $45 \%$ do valor energético da dieta diária. Alguns estudos com este modelo de dieta apontam resultados de melhora dos seguintes marcadores: concentração de glicose, níveis de insulina, sensibilidade à insulina, perda de massa corporal, diminuição da adiposidade e dos triglicerídeos, além de redução ou eliminação do uso de medicamentos, quando comparada à dieta tradicional recomendada pela ADA (DAVIES et al., 2018).

Considerando as informações supracitadas, o objetivo do presente estudo foi avaliar os resultados de ensaios clínicos randomizados, que utilizaram dietas de baixo consumo de carboidratos, em comparação às dietas tradicionais aconselhadas no tratamento de diabetes mellitus tipo 2, quanto aos marcadores de controle da doença, redução ou exclusão do uso de medicamentos e perda de massa corporal.

\section{MÉTODOS}

A revisão narrativa foi realizada utilizando periódicos internacionais de ensaios clínicos randomizados, disponíveis na base de dados Pubmed (https:// www.ncbi.nlm.nih.gov/pubmed/). Pesquisou-se pelo seguinte conjunto de palavras-chave: "low carbohydrate diet" e "type 2 diabetes", em diferentes combinações, e foram considerados estudos publicados nas línguas inglesa, espanhola ou portuguesa no período de 1998 a 2018.

Os estudos selecionados compararam uma dieta de baixo teor de carboidrato, conhecida como "low carb", com outras dietas empregadas no tratamento do diabetes mellitus tipo 2. Considerou-se uma dieta de baixo teor de carboidrato, aquelas que ofereciam percentuais do macronutriente iguais ou inferiores a $45 \%$ ou menos de $130 \mathrm{~g}$ por dia, valores mínimos estabelecidos pelas DRI (Dietary Reference Intakes) (INSTITUTE OF MEDICINE, 2005).

Foram contemplados na presente revisão os estudos que apresentassem os resultados da dieta low carb nos seguintes parâmetros: marcadores bioquímicos de controle da doença (glicemia de jejum, hemoglobina glicada ( $\mathrm{HbA1c}$ ), triglicerídeos e colesterol total e frações), redução ou exclusão do uso de medicamentos, diminuição da circunferência da cintura e perda de massa corporal.
A busca bibliográfica utilizando os termos combinados "low carbohydrate diet" e "type 2 diabetes", resultou em 733 artigos, dos quais foram excluídos aqueles de caráter observacional, os duplicados, os que não possuíam o texto completo para consulta gratuita e os que utilizaram modelos animais, restando 106 artigos. Após a leitura dos títulos e resumos, foram eliminados os artigos com texto elusivo ou com informações incompletas, assim como os que prescreveram quantidades de carboidrato acima do percentual considerado "low carb" pelo presente estudo, finalizando em 47 artigos. Foram excluídos, ainda, os ensaios clínicos não randomizados e sem avaliação de biomarcadores. Após a aplicação de todos os critérios de inclusão, 14 artigos foram selecionados para comporem esta revisão.

A população dos 14 estudos apresentados na Tabela 1 foi caracterizada por 62,2\% de mulheres. Todos os participantes eram maiores de 18 anos, com média de idade de 58 anos, sendo a maior idade registrada igual a 82 anos. A maior parte da população estudada foi classificada como obesa ou acima da massa corporal ideal, com média de IMC de $35,9 \mathrm{~kg} / \mathrm{m}^{2}$.

\section{RESULTADOS}

Quanto à composição e à distribuição dos macronutrientes nas dietas com baixo teor de carboidratos, cinco trabalhos utilizaram a quantidade em gramas por dia (artigos 1, 2, 3, 10 e 11), tendo um único trabalho com orientação menor que 20 gramas por dia (MCAULEY et al., 2006), e os outros com indicação diária entre 20 e 50 gramas de carboidratos (HALLBERG et al., 2018, SASLOW et al., 2017 MCKENZIE et al., 2017, WESTMAN et al., 2008).

A respeito dos resultados das intervenções, entre todos os estudos selecionados apenas um, realizado em 2010, comparou ambas as dietas em conjunto com um treinamento físico resistido (WYCLERLEY et al., 2010). Este estudo foi o que promoveu a maior perda de massa corporal no grupo de dieta low carb adicionada de exercício, sendo esta de $12,8 \%$. Outros três estudos, identificados na Tabela 1 como 1,6 e 10 , também apresentaram perda de massa corporal maior que $10 \%$.

Já no estudo número 4, publicado em 2016, apesar de não haver diferença estatisticamente significativa para a mudança na composição corporal, quando comparado ao grupo controle, o grupo da intervenção atingiu a média de perda ponderal de $9,8 \%$ e $100 \%$ de atenuação do diabetes, contra $33 \%$ do grupo controle. Os artigos 8 e 14 apresentaram 
média de perda de massa corporal também considerada insignificante, sendo de $2,4 \%$ e $1 \%$, respectivamente. 0 artigo 8 , de 2011 , além da pequena perda de massa corporal, também não demonstrou resultados conclusivos para os marcadores de glicemia em jejum e HbA1c. Já o último artigo citado, de 1998, mesmo com a pequena mudança na massa corporal, teve grandes reduções dos parâmetros de glicemia de jejum e $\mathrm{HbA1c}$, além de uma diminuição do uso de medicamentos para diabetes.

Este desfecho importante, de redução ou descontinuação do uso de medicamentos hipoglicemiantes (metformina, sulfonilureia, inibidores da dipeptidil peptidase-4, insulina, entre outros) nos indivíduos que sofreram a intervenção de dieta low carb, após pelo menos dez semanas, foi demonstrado nos dez estudos a seguir: $1,2,3,4,5,6,7,8,10$ e 14 . Já o estudo 9 demonstrou que ambos os grupos, em uso de dieta low carb e recebendo dieta convencional, reduziram o uso de medicamentos, não apresentando diferença significativa. Nos artigos 11, 12 e 13 não foram mencionados resultados sobre o uso de medicamentos anterior à pesquisa e nem ao final da mesma.

Ainda com relação ao manejo do diabetes, os artigos 1, 3, 9 e 14 apresentaram os melhores resultados na redução da glicemia de jejum, com quedas superiores a $15 \%$. Já as concentrações $\mathrm{HbA} 1 \mathrm{c}$ diminuíram mais de $10 \%$ nos grupos em uso de dietas de baixo consumo de carboidratos dos artigos 1, 3, 9, 10 e 14. Dentre estes, os estudos 1 e 9 apresentaram reversão do diabetes. Vale ressaltar que o artigo 13 também apresentou reversão da doença, ainda que a redução da $\mathrm{HbA} 1 \mathrm{c}$ tenha sido menos expressiva. Os artigos 3 e 5 encontraram ainda valores de $\mathrm{HbA1c}$ pós-intervenção low carb de 6,6\%, próximo aos valores iniciais de diagnóstico do diabetes.

Em nenhum trabalho houve registros de eventos hiperglicêmicos graves, que necessitassem de intervenção médica, ou qualquer relato de cetoacidose diabética. Os registros mais frequentes de efeitos colaterais foram: cefaleia, insônia e distúrbios gastrointestinais, como constipação e diarreia.

A Tabela 1 sintetiza as principais características dos ensaios clínicos utilizados.

\section{DISCUSSÃO}

O presente trabalho observou que a maioria dos portadores de diabetes mellitus tipo 2 apresentou sobrepeso ou obesidade. Sabe-se que nesta situação, com o acúmulo de gordura visceral, a necessidade de secreção de insulina se faz aumentada. Sua captação hepática e a eficiência da utilização pelas células, no entanto, estão reduzidas, caracterizando um quadro de resistência periférica à insulina (HALLBERG et al., 2018). Saslow et al. (2017 afirmam que, com a promoção da perda de massa corporal e sua manutenção, alcançadas por mudanças no estilo de vida e adesão a uma dieta baixa em carboidratos, a doença é efetivamente controlada.

\section{Perda de massa corporal}

Esta revisão de artigos comparando dietas convencionais com dietas de baixo consumo de carboidratos em indivíduos diagnosticados com diabetes mellitus tipo 2, demonstrou que as dietas low carb são mais eficazes em promover maior perda de massa corporal. Explicações possíveis para que isto ocorra é a restrição mais rigorosa do consumo de carboidratos ou, ainda, a combinação desta dieta com exercícios físicos (WESTMAN et al., 2008).

Um estudo produzido em 2010, que teve o registro de maior redução de massa corporal dentre as pesquisas aqui avaliadas, observou a aplicação de quatro tipos de intervenções. Um grupo recebeu uma dieta convencional para tratar diabetes, outro foi orientado a consumir uma dieta low carb e os outros dois grupos receberam estas mesmas orientações acompanhadas de um treinamento resistido. Desta forma os autores puderam concluir que, após 16 semanas, um consumo mais baixo em carboidratos, juntamente com o exercício físico, foi a alternativa de tratamento mais eficaz para a diminuição da massa corporal com preservação de massa muscular (WYCHERLEY et al., 2010). Westman et al. (2008) relacionaram tais feitos à substituição de alguns carboidratos da dieta pelo consumo aumentado de gordura e proteína. Com uma intervenção apenas dietética, outros trabalhos obtiveram também perda de massa corporal superior a $10 \%$, a qual esteve associada à restrição severa no consumo de carboidratos, sendo esta menor que 50 gramas ao dia (HALLBERG et al., 2018; SASLOW et al., 2017; TAY et al., 2014; WESTMAN et al., 2008).

Os estudos que apresentaram resultados de pequena perda de massa corporal, ou diferença não significativa nesta medida no grupo low carb, em comparação com o grupo controle, coincidem com aqueles que prescreveram quantidades superiores de carboidratos, sendo igual ou maior que $25 \%$ e chegando até $43 \%$ do valor energético total diário (ROCK et al., 2014). Tal achado é corroborado por Stentz et al. (2016), os quais dividiram os 24 participantes em dois grupos randomizados durante 24 meses, e um deles 
Tabela 1 - Características dos ensaios clínicos com dietas de baixo teor de carboidratos publicados de 1998 a 2018, após aplicação dos critérios de exclusão

\begin{tabular}{|c|c|c|c|c|c|c|c|c|c|}
\hline & \multirow[b]{2}{*}{ Título do artigo e DOI } & \multirow[b]{2}{*}{$\begin{array}{l}\text { Primeiro } \\
\text { autor }\end{array}$} & \multirow[b]{2}{*}{ Ano } & \multirow[b]{2}{*}{ Amostra } & \multirow[b]{2}{*}{$\begin{array}{l}\text { Seguimento } \\
\text { (duração) }\end{array}$} & \multirow[b]{2}{*}{ Desfecho } & \multicolumn{3}{|c|}{ Características da dieta } \\
\hline & & & & & & & $\begin{array}{c}\text { Carboi- } \\
\text { drato }\end{array}$ & Proteína & Gordura \\
\hline 1 & $\begin{array}{l}\text { Effectiveness and Safety of a Novel Care } \\
\text { Model for the Management of Type } 2 \\
\text { Diabetes at } 1 \text { Year: An Open-Label, Non- } \\
\text { Randomized, Controlled Study } \\
\text { DOI: } 10.1007 / \text { s13300-018-0373-9 }\end{array}$ & $\begin{array}{l}\text { Hallberg, } \\
\text { S. J. }\end{array}$ & 2018 & 349 & 12 meses & \begin{tabular}{|l|} 
Maior perda ponderal, \\
redução do uso de \\
medicamentos, reduções \\
de glicemia de jejum \\
e HbA1c, níveis de \\
remissão de diabetes.
\end{tabular} & $<30 \mathrm{~g} / \mathrm{d}$ & $1,5 \mathrm{~g} / \mathrm{kg}$ & SD \\
\hline 3 & $\begin{array}{l}\text { A Novel Intervention Including Individualized } \\
\text { Nutritional Recommendations Reduces } \\
\text { Hemoglobin A1c Level, Medication Use, and } \\
\text { Weight in Type } 2 \text { Diabetes } \\
\text { DOI: } 10.2196 / \text { diabetes.6981 } \\
\end{array}$ & $\begin{array}{l}\text { McKenzie, } \\
\text { A. L. }\end{array}$ & 2017 & 262 & 10 semanas & $\begin{array}{l}\text { Redução de HbA1c e do } \\
\text { uso de medicamentos e } \\
\text { perda ponderal. }\end{array}$ & $<30 \mathrm{~g} / \mathrm{d}$ & $1,5 \mathrm{~g} / \mathrm{kg}$ & SD \\
\hline 4 & $\begin{array}{l}\text { Remission of pre-diabetes to normal glucose } \\
\text { tolerance in obese adults with high protein } \\
\text { versus high carbohydrate diet: randomized } \\
\text { control trial } \\
\text { DOI: } 10.1136 / \text { bmjdrc-2016-000258 }\end{array}$ & Stentz, F. B. & 2016 & 24 & 24 meses & $\begin{array}{l}\text { Redução do uso de } \\
\text { medicamentos. }\end{array}$ & $40 \%$ & $30 \%$ & $30 \%$ \\
\hline 5 & $\begin{array}{l}\text { Weight Loss, Glycemic Control, and } \\
\text { Cardiovascular Disease Risk Factors in } \\
\text { Response to Differential Diet Composition in } \\
\text { a Weight Loss Program in Type } 2 \text { Diabetes: A } \\
\text { Randomized Controlled Trial } \\
\text { DOI: } 10.2337 / \text { dc13-2900 }\end{array}$ & Rock, C. L. & 2014 & 227 & 12 meses & $\begin{array}{l}\text { Redução de glicemia } \\
\text { de jejum, HbA1c e } \\
\text { triglicerídeos. }\end{array}$ & $45 \%$ & $25 \%$ & $30 \%$ \\
\hline 6 & $\begin{array}{l}\text { A Very Low-Carbohydrate, Low-Saturated Fat Diet for } \\
\text { Type } 2 \text { Diabetes Management: A Randomized Trial } \\
\text { DOI: } 10.2337 / \text { dc14-0845. }\end{array}$ & Tay, J. & 2014 & 93 & 24 semanas & $\begin{array}{c}\text { Grande perda de massa } \\
\text { corporal, redução do uso } \\
\text { de medicamentos. }\end{array}$ & $14 \%$ & $28 \%$ & $58 \%$ \\
\hline 7 & $\begin{array}{l}\text { Advice to follow a low-carbohydrate diet has a } \\
\text { favourable impact on low-grade inflammation } \\
\text { in type } 2 \text { diabetes compared with advice to } \\
\text { follow a low-fat diet } \\
\text { DOI: } 10.3109 / 07853890.2014 .894286 \text {. }\end{array}$ & Jonasson, L. & 2014 & 61 & 6 meses & $\begin{array}{l}\text { Redução do uso de } \\
\text { medicamentos. }\end{array}$ & $20 \%$ & SD & SD \\
\hline 8 & $\begin{array}{l}\text { The effect of high-protein, low-carbohydrate } \\
\text { diets in the treatment of type } 2 \text { diabetes: a } 12 \\
\text { month randomised controlled trial } \\
\text { DOI: } 10.1007 / \text { s00125-010-2027-y. } \\
\end{array}$ & Larsen, R. N. & 2011 & 99 & 12 meses & $\begin{array}{l}\text { Redução do uso de } \\
\text { medicamentos. }\end{array}$ & $40 \%$ & $30 \%$ & $30 \%$ \\
\hline 9 & $\begin{array}{l}\text { A High-Protein Diet with Resistance Exercise } \\
\text { Training Improves Weight Loss and Body } \\
\text { Composition in Overweight and Obese } \\
\text { Patients with Type } 2 \text { Diabetes } \\
\text { DOI: } 10.2337 \text { / dc09-1974. }\end{array}$ & $\begin{array}{c}\text { Wycherley, } \\
\text { T. P. }\end{array}$ & 2010 & 59 & 16 semanas & $\begin{array}{l}\text { Reduções de glicemia } \\
\text { de jejum e hemoglobina } \\
\text { glicada, níveis de } \\
\text { remissão de diabetes. }\end{array}$ & $43 \%$ & $33 \%$ & $22 \%$ \\
\hline 10 & $\begin{array}{l}\text { The effect of a low-carbohydrate, ketogenic } \\
\text { diet versus a low-glycemic index diet on } \\
\text { glycemic control in type } 2 \text { diabetes mellitus } \\
\text { DOI: } 10.1186 / 1743-7075-5-36\end{array}$ & $\begin{array}{l}\text { Westman, } \\
\text { E. C. }\end{array}$ & 2008 & 50 & 24 semanas & $\begin{array}{l}\text { Grande perda de massa } \\
\text { corporal, redução do uso } \\
\text { de medicamentos. }\end{array}$ & $>20 \mathrm{~g} / \mathrm{d}$ & SD & SD \\
\hline 11 & $\begin{array}{l}\text { Long-term effects of popular dietary } \\
\text { approaches on weight loss and features of } \\
\text { insulin resistance } \\
\text { DOI: } 10.1038 / \text { sj.ijo. } 0803075\end{array}$ & $\begin{array}{l}\text { McAuley, } \\
\text { K. A. }\end{array}$ & 2006 & 93 & 12 meses & $\begin{array}{c}\text { Redução da } \\
\text { circunferência da } \\
\text { cintura e dos níveis de } \\
\text { triglicerídeos e insulina } \\
\text { no grupo com dieta } \\
\text { hiperproteica. } \\
\end{array}$ & $20-50 \mathrm{~g}$ & SD & SD \\
\hline 12 & $\begin{array}{l}\text { Effect of a High-Protein, Low-Carbohydrate } \\
\text { Diet on Blood Glucose Control in People with } \\
\text { Type } 2 \text { Diabetes } \\
\text { DOI: } 10.2337 / \text { diabetes.53.9.2375 } \\
\end{array}$ & $\begin{array}{l}\text { Gannon, } \\
\text { M. C. }\end{array}$ & 2004 & 8 & 5 semanas & $\begin{array}{l}\text { Redução de } \\
\text { hemoglobina glicada e } \\
\text { glicemia de jejum. }\end{array}$ & $20 \%$ & $30 \%$ & $50 \%$ \\
\hline 13 & $\begin{array}{l}\text { Effect of a High-Protein, High- } \\
\text { Monounsaturated Fat Weight Loss Diet on } \\
\text { Glycemic Control and Lipid Levels in Type } 2 \\
\text { Diabetes } \\
\text { DOI: } 10.2337 / \text { diacare.25.3.425 }\end{array}$ & Parker, B. & 2002 & 54 & 12 semanas & $\begin{array}{l}\text { Redução de } \\
\text { hemoglobina glicada, } \\
\text { níveis de remissão de } \\
\text { diabetes. }\end{array}$ & $42 \%$ & $28 \%$ & $27 \%$ \\
\hline 14 & $\begin{array}{l}\text { Utility of a short-term } 25 \% \text { carbohydrate } \\
\text { diet on improving glycemic control in type } 2 \\
\text { diabetes mellitus } \\
\text { DOI: } 10.1080 / 07315724.1998 .10718808\end{array}$ & $\begin{array}{c}\text { Gutierrez, } \\
\text { M. }\end{array}$ & 1998 & 9 & 20 semanas & \begin{tabular}{|c|} 
Redução do uso de \\
medicamentos, reduções \\
de glicemia de jejum e \\
hemoglobina glicada.
\end{tabular} & $25 \%$ & $30 \%$ & $45 \%$ \\
\hline
\end{tabular}


recebeu uma dieta com $40 \%$ de carboidratos. Não foi encontrada diferença estatisticamente significativa na perda ponderal, no entanto os autores concluíram que uma dieta low carb beneficiou $100 \%$ dos participantes do estudo com a reversão do diabetes mellitus.

Larsen et al. (2011) e Wyclerley et al. (2010) relataram uma pequena porcentagem de perda de massa corporal ao final de seu estudo. Este trabalho, no entanto, apresenta limitações, pois afirma que alguns dos participantes descontinuaram o tratamento, sendo a maior parte do grupo de dieta low carb, mas retornaram para reavaliações de seguimento, o que pode ter interferido nos resultados de perda de massa corporal. Além disso, o controle de adesão à dieta foi realizado por relatos dos próprios participantes, que autogerenciavam a sua ingestão alimentar, o que também pode incorrer em erro.

Quanto ao estudo produzido em 1998 por Gutierrez et al., a perda de massa corporal verificada no grupo de dieta low carb também foi considerada não significativa. Este resultado pode ser explicado pelo fato de os participantes terem retomado um consumo de carboidratos de $55 \%$ até o final do estudo, ou seja, eles foram reintroduzidos a uma dieta normoglicídica após as primeiras oito semanas.

Os benefícios da promoção de perda de massa corporal, com a manutenção da massa muscular, conforme demostram estes experimentos, estão relacionadas à melhora do perfil metabólico de insulina em jejum e HbA1c (WESTMAN et al., 2008; GOMEZ-ARBELAEZ et al., 2017).

\section{Reduções de HbA1c}

Em relação à redução de $\mathrm{HbA1c}$, a maioria dos trabalhos apresentaram-na como desfecho primário. Hallberg et al. (2018) e Saslow et al. (2017 demostraram melhorias significativas em apenas 70 dias de intervenção, atingindo reduções expressivas de HbA1c. Esta diminuição foi associada a uma melhora da inflamação e da função hepática, atingindo condições normais de glicemia.

Saslow et al. (2017) e Mckenzie et al. (2017) compararam uma dieta cetogênica sem restrição calórica com uma dieta convencional aconselhada pela ADA (American Diabetes Association) em adultos com pré-diabetes ou já diagnosticados com diabetes mellitus. Dentre os participantes do grupo controle, nenhum alcançou valores para $\mathrm{HbA} 1 \mathrm{c}$ abaixo de 5,7\%, o que foi alcançado por $13 \%$ do grupo da dieta cetogênica, após os seis primeiros meses. Outros $56 \%$ da amostra em dieta cetogênica atingiram uma redução de $0,5 \%$ da HbA1c, comparado a $22 \%$ do grupo controle.

Mckenzie et al. (2017) e Stentz et al. (2016) também encontraram resultados positivos de redução da HbA1c e da glicemia em jejum ao prescreverem dieta hiperproteica $(1,5 \mathrm{~g} / \mathrm{kg}$ de massa corporal) e ingestão de carboidratos abaixo de $30 \mathrm{~g}$ por dia. Entre 10 e 11 semanas de intervenção, 56\% dos participantes já haviam atingido níveis inferiores a 6,5\% para $\mathrm{HbA1c}$, dos quais $26 \%$ (39 participantes) alcançaram tal feito sem o uso de medicamentos para o controle da doença. Nos estudos de Rock et al. e Tay et al., publicados em 2014, 71\% dos participantes do grupo de intervenção reduziram seus valores de HbA1c a uma média de $6,9 \%$, contra $7,5 \%$ do grupo controle.

0 estudo de Parker et al. (2002) também promoveu reduções na $\mathrm{HbA1c}$ e relatou que, ao fim das 12 semanas de intervenção, os participantes haviam se tornado mais sensíveis à insulina. Gutierrez et al. (1998) verificaram que os indivíduos em low carb quando colocados em uma dieta com $55 \%$ de carboidratos, aumentaram significativamente os níveis de $\mathrm{HbA1c}$, piorando o controle glicêmico que haviam conseguido com a restrição de carboidratos. Estes achados sugerem que, para obtenção dos resultados esperados, a dieta low carb deve ser realizada sem interrupção por um longo período.

Sabe-se que a HbA1c é um marcador retrospectivo que refere a glicemia média nos últimos 2 a 3 meses e que corresponde ao tempo de meia-vida das hemácias. Clinicamente são considerados os valores de $4 \%$ a $6 \%$ como normais, e acima de $7 \%$ é maior a associação com as doenças crônicas não transmissíveis (DCNTs), (SBD, 2018).

As melhoras metabólicas, com reduções expressivas de HbA1c, chegando a níveis de reversão da doença, podem explicar a diminuição ou eliminação do uso de medicamentos. Vale ressaltar que se considerou como reversão quando algum paciente, após 6 meses de tratamento, atingia níveis de glicemia em jejum menor que $100 \mathrm{mg} / \mathrm{dL}$ e menor que $140 \mathrm{mg} /$ dL após 2 horas da refeição (ROCK et al., 2014).

\section{Redução ou descontinuação do uso de medicamentos}

Hallberg et al. (2018) e Saslow et al. (2017) concluíram, após os 12 meses de intervenção, que a expressiva maioria dos participantes do grupo em dieta low carb interrompeu ou diminuiu o uso de insulina bem como de outros medicamentos. Achados 
semelhantes foram observados em diversas outras pesquisas que compararam a dieta reduzida em glicídios com as recomendações normoglicídicas e recomendadas pela ADA (MCKENZIE et al., 2017; LARSEN et al., 2011; ROCK et al., 2014; TAY et al., 2014; JONASSON et al., 2014).

Da mesma maneira, Westman et al. (2008) concluíram que o uso de medicação também foi descontinuado ou reduzido em maior proporção no grupo de dieta low carb, neste caso cetogênica, e a redução da utilização de insulina também foi maior. Uma investigação importante neste trabalho foi que os autores não apenas compararam uma dieta low carb com uma de aconselhamento tradicional para indivíduos com diabetes mellitus tipo 2, mas se atentaram aos valores de índice glicêmico de ambas as dietas, sendo uma dieta low carb cetogênica, com menos de $20 \mathrm{~g}$ de carboidratos por dia, e outra com $55 \%$ do valor energético total proveniente de carboidratos de baixo índice glicêmico. Neste trabalho é possível observar os macronutrientes não apenas de forma quantitativa, mas também qualitativa, considerando a velocidade de absorção da glicose.

Desta forma, alimentos que apresentam liberação mais lenta e gradual de glicose são considerados de baixo índice glicêmico, ricos em fibras, e possuem melhor controle da liberação de insulina pelo organismo, mantendo a glicose sanguínea dentro dos valores normais, sem quadros de hiperglicemia. Já os alimentos de alto índice glicêmico, quando comparados com o alimento-controle, o pão branco, possuem resposta similar a este, tendo uma liberação rápida de glicose no sangue, promovendo uma maior produção e liberação de insulina, como uma pior resposta fisiológica, quando comparado aos alimentos de baixo índice glicêmico (SBD, 2019).

Os resultados apresentados por Westman et al. (2008), contudo, mostraram que uma dieta low carb ainda foi mais efetiva no controle do diabetes mellitus tipo 2 do que uma dieta normoglicídica de baixo índice glicêmico.

Desta forma, dez trabalhos encontraram diminuição do uso de medicamentos, com taxas variando de $20 \%$ até $100 \%$. Os autores justificam que as maiores taxas de redução do uso de medicamentos devem-se ao menor consumo de carboidratos na dieta, com melhor controle da glicemia e provável melhora da sensibilidade à insulina. Já os menores índices podem estar relacionados às características de uma amostra reduzida e um menor tempo de intervenção.
Isto concorda com o trabalho feito por Gutierrez et al. (1998), que demonstrou que os pacientes que não haviam conseguido êxito no tratamento com sulfonilureia, quando em uma dieta baixa em carboidratos, tendiam a reduzir a necessidade do uso de insulina. Resultados semelhantes foram vistos por Jonasson et al. (2014). Apesar de não observarem alteração no uso de medicação oral pelos participantes da sua pesquisa, a dose total de insulina utilizada foi reduzida significativamente no grupo que praticou a dieta low carb.

\section{Insulina de jejum}

Os achados de Stentz et al. (2016) e Rock et al. (2014) foram favoráveis para a redução de insulina de jejum, demonstrando que os indivíduos em low carb voltaram a apresentar uma tolerância normal à glicose. Nos estudos de Wycherley et al. (2010) e Westman et al. (2008), a intervenção de dieta low carb com acréscimo de exercício resistido apresentou o dobro na redução de insulina em jejum, quando comparada aos outros grupos. Tal achado já foi bastante justificado pelo efeito do exercício físico na captação muscular da glicose, podendo sensibilizar os receptores intracelulares, como as vias de sinalização IR, IRS, PI3-k e Akt, melhorando a captação de glicose em vias independentes de insulina, resposta adaptativa à prática regular de exercício físico (BARROS et al., 2019). Desta forma o exercício melhora a resistência à insulina, que está associada à obesidade, contribuindo, ain$\mathrm{da}$, com outras complicações, como as dislipidemias, que estão altamente associadas aos níveis glicêmicos descompensados (BARBOSA; GOMES; PALMA, 2017).

\section{Triglicérides e menor risco de doenças cardiovasculares}

Considerando que portadores de diabetes têm um risco aumentado para doenças cardiovasculares e nefropatias, é notável a redução dos marcadores associados a estas complicações. Alguns trabalhos evidenciaram como desfecho secundário diminuições de triglicérides, LDL-colesterol e o aumento de HDL-colesterol, sendo estas mudanças mais efetivas naqueles participantes com distúrbios metabólicos mais agravados (MCKENZIE et al., 2017; STENTZ et al., 2016; TAY et al., 2014).

Vale mencionar que, em alguns estudos, a prática de uma dieta restrita em carboidratos não foi isenta de efeitos adversos. Relatos de cefaleia, insônia e distúrbios gastrointestinais, como constipação 
e diarreia, foram registrados por autores que avaliaram os efeitos deste padrão de consumo alimentar (BOSTOCK et al., 2020).

\section{CONCLUSÃO}

Em favor do que se afirma na literatura, uma dieta com o consumo mais baixo de carboidratos parece ser mais efetiva no controle do diabetes mellitus tipo 2, chegando, em alguns casos, a níveis de reversão da doença. Este modelo de alimentação pode garantir ao paciente um melhor controle glicêmico, com diminuição expressiva de glicose sérica em jejum, HbA1c, além de maior redução de massa corporal e o uso de medicamentos. As vantagens estendem-se também aos marcadores de risco cardiovascular, como redução de triglicérides e LDL-colesterol e aumento do HDL-colesterol, promovendo redução no risco de doença cardiovascular.

Deve-se considerar como possível desvantagem a dificuldade de adesão do paciente à restrição severa de carboidratos, além de ser preciso enfatizar a necessidade de acompanhamento para que não haja depleção de massa muscular e agravo de efeitos colaterais.

Ainda são necessários mais estudos, com amostra aumentada, para que se confirmem os achados e que as diretrizes apresentem a dieta low carb como uma estratégia eficaz para o manejo do diabetes mellitus tipo 2.

\section{REFERÊNCIAS}

ADA. American Diabetes Association. Diagnosis and classification of diabetes mellitus. Diabetes Care, v. 37, Supplement 1, p. S81-S90, 2014.

ADA. American Diabetes Association. Standards of medical care in diabetes: 2017. Diabetes Care, v. 40, Supplement 1 , p. S1-S135, 2017.

ABESO. Associação Brasileira Para o Estudo da Obesidade e da Síndrome Metabólica. Diretrizes brasileiras de obesidade. 4. ed. São Paulo, 2016.

BARBOSA, V. S. N.; GOMES, L. S.; PALMA, D. C. A. Dislipidemia em pacientes com diabetes tipo 2. Saúde e Pesqui., v. 10, n. 3, p. 579-585, 2017.

BARROS, L. S.; NUNES, C. C. A influência do exercício físico na captação de glicose independente de insulina. HU Rev., v. 45, n. 1, p. 59-64, 2019.

BASU, S. et al. Relationship of sugar to population-level diabetes prevalence: an econometric analysis of repeated cross-sectional data. PLoS One, v. 8, n. 2, p. 1-8, 2013.

BOSTOCK, E. C. S. et al. Consumer Reports of "Keto Flu" Associated With the Ketogenic Diet. Front Nutr., v. 7, n. 20, 2020.
DAVIES, M. J. et al. Management of hyperglycaemia in type 2 diabetes, 2018. A consensus report by the American Diabetes Association (ADA) and the European Association for the Study of Diabetes (EASD). Diabetes Care, 61(12), p. 2.461-2.498, 2018.

GANNON, M. C.; NUTTALL, F. Q. Effect of a high-protein, low-carbohydrate diet on blood glucose control in people with type 2 diabetes. Diabetes, v. 53, n. 9, p. 2.375-2.382, 2004.

GOMEZ-ARBELAEZ, D. et al. Body Composition Changes After Very-Low-Calorie Ketogenic Diet in Obesity Evaluated by 3 Standardized Methods. J Clin Endocrinol Metab., v. 102, n. 2, p. 488-498, 2017.

GROSS, J. L. et al. Diabetes melito: diagnóstico, classificação e avaliação do controle glicêmico. Arq. Bras. Endocrinol. Metab., São Paulo, v. 46, n. 1, p. 16-26, 2002.

GUTIERREZ, M. et al. Utility of a short-term $25 \%$ carbohydrate diet on improving glycemic control in type 2 diabetes mellitus. Journal of the American College of Nutrition, v. 17, p. 595-600, 1998.

HALLBERG, S. J. et al. Effectiveness and safety of a novel care model for the management of type 2 diabetes at 1 year: an open-label, non-randomized, controlled study. Diabetes Ther, 9(2), p. 583-612, 2018. DOI: 10.1007/s13300018-0373-9.

INSTITUTE OF MEDICINE. Dietary reference intakes for energy, carbohydrate, fiber, fat, fatty acids, cholesterol, protein, and amino acids. Washington, DC: National Academy Press; 2005.

IDF. International Diabetes Federation. The latest edition of the IDF Diabetes Atlas. 2019. Disponivel em: https://www. idf.org/e-library/epidemiology-research/diabetes-atlas/ 159-idf-diabetes-atlas-ninth-edition-2019.html. Acesso em: 26 jun. 2020.

JONASSON, L. et al. Advice to follow a low-carbohydrate diet has a favourable impact on low-grade inflammation in type 2 diabetes compared with advice to follow a low-fat diet. Ann Med., v. 43, n. 3, p. 182-187, 2014.

JOSLIN DIABETES CENTER. Know your food groups with diabetes. Disponível em: https://onlineclasses.joslin.org/info/ know_your_food_groups_with_diabetes.html. Acesso em: 15 jun. $201 \overline{8}$.

LARSEN, R. N. et al. The effect of high-protein, low-carbohydrate diets in the treatment of type 2 diabetes: a 12 month randomised controlled trial. Diabetologia, v. 54, p. 731-740, 2011.

MCAULEY, K. A. et al. Long-term effects of popular dietary approaches on weight loss and features of insulin resistance. International Journal of Obesity, v. 30, n. 13, p. 342349, 2006.

MCKENZIE, A. L. et al. A novel intervention including individualized nutritional recommendations reduces hemoglobin a1c level, medication use, and weight in type 2 diabetes. JMIR Diabetes, v. 2, n. 1, 2017. 
MCLELLAN, K. C. P. et al. Diabetes mellitus do tipo 2, síndrome metabólica e modificação no estilo de vida. Rev. Nutr., Campinas, v. 20, n. 5, p. 515-524, 2007.

PARKER, B. et al. Effect of a high-protein, high-monounsaturated fat weight loss diet on glycemic control and lipid levels in type 2 diabetes. Diabetes Care, v. 25, n. 3, p. 425-430, 2002.

ROCK, C. L. et al. Weight loss, glycemic control, and cardiovascular disease risk factors in response to differential diet composition in a weight loss program in type 2 diabetes: a randomized controlled trial. Diabetes Care, v. 37, n. 6, p. 1.573-1.580, 2014.

SASLOW, L. R. et al. Twelve-month outcomes of a randomized trial of a moderate-carbohydrate versus very low-carbohydrate diet in overweight adults with type 2 diabetes mellitus or prediabetes. Nutrition \& Diabetes, v. 7, n. 304, 2017.

SOARES, A. H. Considerações sobre diabetes e pré diabetes. 2016. Disponível em: http://www.diabetes.org.br/publico/ temas-atuais-sbd/1427-consideracoes-sobre-diabetes-e-pre-diabetes. Acesso em: 10 jan. 2018.

SBD. Sociedade Brasileira de Diabetes. Diretrizes da Sociedade Brasileira de Diabetes 2019-2020. São Paulo: Editora Clannad, 2019.

SBD. Sociedade Brasileira de Diabetes. Tipos de diabetes. 2018. Disponível em: http://www.diabetes.org.br/publico/ diabetes/tipos-de-diabetes. Acesso em: 10 jan. 2018.

SBD. Sociedade Brasileira de Diabetes. Atualização sobre hemoglobina glicada (A1c) para avaliação do controle glicêmico e para o diagnóstico do diabetes: aspectos clínicos e laboratoriais 2017/2018. São Paulo: Editora Clannad, 2017.

SBD. Sociedade Brasileira de Diabetes. Conduta terapêutica no diabetes tipo 2. São Paulo: Editora Clannad, 2019.

SBP. Sociedade Brasileira de Pediatria; Associação Brasileira de Alergia e Imunopatologia. Consenso brasileiro sobre alergia alimentar: 2007. Rev. Med., v. 18, supl. 1, p. S1-S44, 2008.

STENTZ, F. B. et al. Remission of pre-diabetes to normal glucose tolerance in obese adults with high protein versus high carbohydrate diet: randomized control trial. BMJ Open Diabetes Res. Care, v. 4, n. 1, 2016.

TAY, J. et al. A very low-carbohydrate, low - saturated fat diet for type 2 diabetes management: a randomized trial. Diabetes Care, v. 37, n. 11, p. 2.909-2.918, 2014.

VOLEK, S. J. et al. Carbohydrate restriction has a more favorable impact on the metabolic syndrome than a low fat diet. Lipids, v. 44, n. 4, p. 297-309, 2008.

WESTMAN, E. C. et al. The effect of a low-carbohydrate, ketogenic diet versus a low-glycemic index diet on glycemic control in type 2 diabetes mellitus. Nutr. Metab., v. 5, n. 36, p. 1-9, 2008.

WYCLERLEY, T. P. et al. A high-protein diet with resistance exercise training improves weight loss and body composition in overweight and obese patients with type 2 diabetes. Diabetes Care, v. 33, n. 5, p. 969-976, 2010. 